\title{
POISSON SEMIGROUPS AND SINGULAR INTEGRALS
}

\author{
BJÖRN E. J. DAHLBERG
}

\begin{abstract}
Let $D \subset \mathbf{R}^{n}$ be a Lipschitz domain and consider the bilinear form $\int_{D} u(\partial v / \partial y) d P$. We show that the form is bounded if $v$ is harmonic with boundary values in $L^{2}$, if $u$ is smooth with its nontangential maximal function in $L^{2}$ and $\int_{D} \operatorname{dist}\{P, \partial D\}|\operatorname{grad} u|^{2} d P<\infty$.
\end{abstract}

1. Introduction. The purpose of this note is to study the properties of the solution operator that corresponds to the Dirichlet problem of the Laplace operator in Lipschitz domains. That is, we will consider a Lipschitz function $\varphi: \mathbf{R}^{n} \rightarrow \mathbf{R}$; i.e.,

$$
|\varphi(x)-\varphi(z)| \leq M|x-z|,
$$

and with $\varphi$ the associated Lipschitz domain $D=\{(x, y): y>\varphi(x)\}$. Given a function $f$ on $\Gamma=\partial D$, we let $u$ be the harmonic function in $D$ with boundary values $f$. Putting $S(t) f(P)=u(P+(0, t))$, we see that $\{S(t)\}_{t \geq 0}$ is a semigroup; i.e., $S\left(t_{1}+t_{2}\right)=S\left(t_{1}\right) S\left(t_{2}\right)$ for $t_{i} \geq 0$, and $S(0)$ is the identity. A basic fact is now that $\{S(t)\}$ is a semigroup on $L^{2}(\Gamma)$; i.e.,

$$
\sup _{t>0}\|S(t) f\|_{L^{2}(\Gamma)} \leq C(M)\|f\|_{L^{2}(\Gamma)} \quad \text { and } \quad \lim _{t \downarrow 0}\|S(t) f-f\|=0
$$

whenever $f \in L^{2}(\Gamma)$. Here $L^{2}(\Gamma)$ is taken with respect to the surface measure $\sigma$ of $\Gamma$. For a proof of this fact see Dahlberg [D1] and also $\S 2$ of this paper. It now follows from the Hille-Yoshida theorem (see e.g. Yoshida $[\mathbf{Y}]$ ) that the semigroup $\{S(t)\}$ has a generator $A$ on $L^{2}(\Gamma)$ that is densely defined on $L^{2}(\Gamma)$.

We first study the domain of definition $D(A)$ for the generator $A$. To this end we let $L_{1}^{2}(\Gamma)$ denote the class of functions $f \in L^{2}(\Gamma)$ such that the distributional derivatives of $g(x)=f(x, \varphi(x))$ belong to $L^{2}\left(\mathbf{R}^{n}\right)$. Our first result is

$$
D(A)=L_{1}^{2}(\Gamma) \text {. }
$$

The inclusion $D(A) \subset L_{1}^{2}(\Gamma)$ follows from the results by Jerison and Kenig [JK], and the other inclusion follows from area integral estimates from [D2].

We also study the adjoint $\left\{S^{*}(t)\right\}$ of the semigroup $\{S(t)\}$. We find an explicit representation of $\left\{S^{*}(t)\right\}$ by constructing a bounded invertible mapping $T: L^{2}(\Gamma) \rightarrow L^{2}(\Gamma)$ such that

$$
S^{*}(t)=T^{-1} S_{\#}(t) T .
$$

Here $\left\{S_{\#}(t)\right\}$ is the semigroup on $L^{2}(\Gamma)$ that corresponds to solving the Dirichlet problem in $\{(x, y): y<\varphi(x)\}=\mathbf{R}^{n+1}-\bar{D}$. The main step in the proof of (1.3)

Received by the editors June 3, 1985.

1980 Mathematics Subject Classification. Primary 31B25; Secondary $42 B 99$. 
is to show the invertibility of the operator $L=A U$, where $A$ is the generator of $\{S(t)\}$ and $U f$ is the boundary value of the harmonic function

$$
v_{f}(P)=\int_{\Gamma} f(Q)|P-Q|^{2-u} d \sigma(Q), \quad P \in D .
$$

From this it follows that the solution $u$ of the Dirichlet problem with boundary values $f$ can be represented as $u=\partial v_{g} / \partial_{y}$, where $g=L^{-1} f$.

We now use (1.3) for a study of singular integrals of the form

$$
B_{A}(u)=\int_{D} \sum_{k=1}^{n+1} A_{k} \frac{\partial u}{\partial x_{k}} d x
$$

where $u$ is a harmonic function in $D \subset \mathbf{R}^{n+1}$ and $A=\left(A_{1}, \ldots, A_{n+1}\right)$ is a vectorvalued function. The objective here is to estimate $\left|B_{A}(u)\right|$ in terms of the boundary values of $u$ and the behavior of $A_{1}, \ldots, A_{n+1}$. We would like to remark that $B_{A}(u)$ is a version for nonsmooth regions of the paraproduct of Bony; see Meyer $[\mathbf{M}]$.

Integrals of the form (1.4) appear in several different contexts. In Dahlberg [D3] it is pointed out that integrals of this form represent the variation of the solution of the Dirichlet problem when the coefficients of the differential equation vary. In [D4] this was used to study the solvability of the Dirichlet problem for uniformly elliptic second order partial differential equations in selfadjoint form.

In Dahlberg, Kenig and Verchota [DKV] it is shown that the study of the Dirichlet problem for the biharmonic equation in Lipschitz domains also leads to singular integrals of the form (1.4).

We recall that a domain $D$ is called a Lipschitz domain if $D$ and $\partial D$ can locally be described by graphs of Lipschitz functions. For surveys of the properties of harmonic functions in Lipschitz domains we refer to the surveys in Dahlberg [D4] and Kenig $[\mathbf{K}]$. For other aspects of the connection between singular integrals and boundary value problems we refer to the survey in Calderon $[\mathbf{C}]$.

In Dahlberg [D1, D2] it is shown that if $D$ is a bounded Lipschitz domain and if $\Delta u=0$ in $D$ with $u=f$ on $\partial D$ then

$$
\|M(u)\|_{L^{2}(\Gamma)} \leq C\|f\|_{L^{2}(\Gamma)}
$$

where the maximal function $M(u)$ is defined by $M(u)(P)=\sup \{|u(Q)|: Q \in D$, $|Q-P|<2 \operatorname{dist}\{Q, \partial D\}\}, P \in \partial D$. Putting $\delta(Q)=\operatorname{dist}\{Q, \partial D\}$, we also have the estimate

$$
\int_{D} \delta|\nabla u|^{2} d P \leq C\|f\|_{L^{2}(\Gamma)}^{2},
$$

where $\nabla u$ is the gradient of $u$. Estimates (1.5) and (1.6) motivate the introduction of the norm

$$
\|\mid u\|=\left(\int_{\partial D}(M(u))^{2} d \sigma\right)^{1 / 2}+\left(\int_{D} \delta|u|^{2} d P\right)^{1 / 2} .
$$

The above estimate can be summarized as follows: If $u$ is harmonic with boundary values $f$, then

$$
\|u\| \mid \leq C\|f\|_{L^{2}(\Gamma)}
$$


Our main result is that if $A$ is a smooth vector-valued function in $D$ with $\|\mid A\| \|<\infty$, then for all harmonic functions $u$ in a bounded Lipschitz domain we have the estimate

$$
\left|B_{A}(u)\right| \leq C(D)\left|\|A \mid\|\|M(u)\|_{L^{2}(\Gamma)} .\right.
$$

2. The generator for the Poisson semigroup. We will begin by discussing the $L^{2}$-estimates for area integrals and maximal functions. We put $D=\{(x, y): y>$ $\varphi(x)\}$, where $\varphi$ is a real-valued Lipschitz function. In what follows we do not explicitly mention that the constants in our estimates depend on the dimension.

THEOREM 1. Suppose $f \in L^{2}(\Gamma), \Gamma=\partial D$. Then there is a harmonic function $u$ in $D$ with $M(u) \in L^{2}(\Gamma)$ such that $u$ converges to $f$ nontangentially a.e. Furthermore, $\int_{D} \delta(P)|\nabla u|^{2} \leq C\|f\|_{L^{2}(\Gamma)}^{2}$, where $\delta(P)=\operatorname{dist}(P, \Gamma)$ and $\|M(u)\|_{L^{2}(\Gamma)} \leq$ $C\|f\|_{L^{2}(\Gamma)}$, where $C$ only depends on the Lipschitz constant of $\varphi$.

ProOF. There is a constant $A>0$ which can be taken to depend only on the Lipschitz constant of $\varphi$ such that $D_{\nu}$ is a starshaped Lipschitz domain wiı̂h Lipschitz constant independent of $\nu$, where $D_{\nu}=\{(x, y): \varphi(x)<y<\varphi(x)+$ $\nu A,|x|<\nu\}, \nu=1,2, \ldots$ Let $u_{\nu}$ solve the Dirichlet problem in $D_{\nu}$ with data $f_{\nu}=f$ on $\partial D \cap \partial D_{\nu}$ and 0 elsewhere on $\partial D_{\nu}$. Letting $M_{\nu}(u)$ be the maximal function of $u_{\nu}$ and putting $M_{\nu}(P)=\operatorname{dist}\left(P, D_{\nu}\right)$, we have from the estimates of the Dirichlet problem for the bounded case that $[$ D1, D2]

$$
\int_{\partial D \cap \partial D_{\nu}}\left(M_{\nu}(u)\right)^{2} d \sigma+\int_{D_{\nu}} \delta_{\nu}(P)\left|u_{\nu}(P)\right|^{2} d P \leq C \int_{\partial D \cap \partial D_{\nu}} f^{2} d \sigma \leq C \int_{\partial D} f^{2} d \sigma .
$$

Letting $\nu \rightarrow \infty$, we see that Theorem 1 follows.

We have the following converse of Theorem 1.

THEOREM 2. Suppose $u$ is harmonic in D. If there is a point $P_{0}=\left(X_{0}, \varphi\left(X_{0}\right)\right)$ $\in \Gamma$ such that $\lim _{y \rightarrow+\infty} u\left(x_{0}, y\right)=0$, then

$$
\int_{\Gamma}(M(u))^{2} d \sigma \leq C \int_{D} \delta(P)|\nabla u(P)|^{2} d P,
$$

where $C$ only depends on the Lipschitz constant of $\varphi$.

ProOF. We may without loss of generality assume that $X_{0}=0$. Putting $P_{\nu}=\left(0, \varphi(0)+\frac{1}{2} \nu A\right)$ and $u_{\nu}(P)=u(P)-u\left(P_{\nu}\right)$, we have from the Lusin area integral estimates in [D2] that

$$
\int_{\partial D \cap \partial D_{\nu}}\left(M_{\nu}\left(u_{\nu}\right)\right)^{2} d \sigma \leq C \int_{D_{\nu}} \delta_{\nu}(P)\left|\nabla u_{\nu}(P)\right|^{2} d P \leq C \int_{D} \delta(P)|\nabla u(P)|^{2} d P .
$$

Letting $\nu \rightarrow \infty$ yields Theorem 2 .

We remark that the above theorems can be generalized to weighted $L^{p}$-spaces by the same reduction to the bounded case. Since we will not use these more general results, we leave these extensions to the reader.

We shall next study the domain of definition of the generator $A$ of the Poisson semigroup. For $f$ a differentiable function on $\Gamma$ let $\nabla_{t} f$ be the (tangential) gradient of $f$. In [DKV] it is shown that if $u$ solves the Dirichlet problem with boundary values $f$, then

$$
\int_{\Gamma}(M(\nabla u))^{2} d \sigma \leq C \int_{\Gamma}\left|\nabla_{t} f\right|^{2} d \sigma
$$


THEOREM 3. Let $D(A)$ denote the domain of definition of the generator $A$ of the Poisson semigroup for the domain $D$. Then a function $f \in L^{2}(\Gamma)$ belongs to $D(A)$ if and only if $\int\left|\nabla_{t} f\right|^{2} d \sigma<\infty$.

PROOF. In view of (2.1) and Theorem 1, it is enough to show that if $u$ is harmonic in $D$ and if $\lim _{y \rightarrow \infty}|u(x, y)|=0$ for all $x \in \mathbf{R}^{n-1}$, then

$$
\int_{D} \delta(P)|\nabla u(P)|^{2} d P \leq C \int_{D} \delta(P)\left(\frac{\partial u}{\partial y}(P)\right)^{2} d P .
$$

To show (2.2) we first observe that

$$
\int_{D} \delta(P)^{3}\left|\nabla \frac{\partial u}{\partial y}\right|^{2} d P \leq C \int_{D} \delta(P)\left(\frac{\partial u}{\partial y}\right)^{2} d P
$$

and since

$$
\frac{\partial u}{\partial x_{j}}(x, y)=-\int_{y}^{\infty} \frac{\partial^{2} u}{\partial x_{j} \partial_{y}}(x, s) d s, \quad 1 \leq j \leq n-1,
$$

(2.2) now follows from Hardy's inequality; see Stein [S, p. 272].

We next need a convenient way of representing the solution of the Dirichlet problem in $D$. For $f \in L^{2}(\Gamma) \cap L^{1}(\Gamma)$ put $S f(P)=\int_{\Gamma} f(Q)|P-Q|^{2-n} d \sigma(Q)$, $P \in \mathbf{R}^{n}-\Gamma$. Then $S f$ is harmonic in $\mathbf{R}^{n}-\Gamma$, and by the boundedness of the Cauchy integral [CMM] it follows that $\nabla S f$ has nontangential limits a.e. on $\Gamma$ and $\|M(\nabla S f)\|_{L^{p}(\Gamma)} \leq C_{p}\|f\|_{L^{p}(\Gamma)}, 1<p<\infty$. Now let

$$
T f(P)=\lim _{s \downarrow 0} \frac{\partial S f}{\partial y}(P+(0, s)) .
$$

THEOREM 4. The operator $T$ is a bounded invertible operator on $L^{2}(\Gamma)$. Furthermore, the norms of $T$ and $T^{-1}$ depend only on the Lipschitz constant of $\varphi$.

PROOF. The boundedness of $T$ follows from the above remarks. To show that $T$ is invertible, we first observe that from (2.2) it follows that $\left\|\nabla_{t} S(f)\right\|_{L^{2}(\Gamma)} \leq$ $C\|T f\|_{L^{2}(\Gamma)}$. Letting $M_{-}$denote the maximal function with respect to $D_{-}=$ $\mathbf{R}^{n}-\bar{D}$, we have from (2.1) and the continuity of $S(f)$ across $\Gamma$ that

$$
\left\|M_{-}(\nabla S(f))\right\|_{L^{2}(\Gamma)} \leq C\left\|\nabla_{t}(S(f))\right\|_{L^{2}(\Gamma)} \leq C\|T f\|_{L^{2}(\Gamma)} .
$$

If $\partial / \partial n$ and $\partial / \partial n_{-}$denote the normal derivatives with respect to $D$ and $D_{-}$, respectively, we have by the well-known jump relations that $\partial S(f) / \partial n+\partial S(f) / \partial n=c f$, where $c \neq 0$ is independent of $\varphi$ and $f$. Hence $c\|f\| \leq\|F f\|$. Since $T$ varies continuously with $\varphi$ and $T=c I, c \neq 0$, when $\varphi=0$, the invertibility of $T$ follows easily.

3. Singular integrals. We shall begin by using the above representation of the solution of the Dirichlet problem. We shall slightly change notation and put for $f \in L^{2}\left(\mathbf{R}^{n-1}\right)$,

(3.1) $T f(x)=\lim _{y \downarrow 0} \int(y+\varphi(x)-\varphi(\xi))\left[|x-\xi|^{2}+(y+\varphi(x)-\varphi(\xi))^{2}\right]^{-n / 2} f(\xi) d \xi$

and let $T_{\#} f(x)$ be the corresponding limit as $y \uparrow 0$; and if $u$ and $u_{\#}$ denote the solutions of the Dirichlet problem with data $f(x)$ for $P=(x, \varphi(x)) \in \Gamma$ in $D$ and $\mathbf{R}^{n}-\bar{D}$, respectively, we put

$$
U(t) f(x)=u(x, \varphi(x)+t), \quad U_{\#}(t) f(x)=u_{\#}(x, \varphi(x)-t) .
$$


For $t>0$ let

$$
V(t) f(x)=\int f(\xi)(t+\varphi(x)-\varphi(\xi))\left(|x-\xi|^{2}+(y+t-\varphi(x))^{2}\right)^{-n / 2} d \xi .
$$

From Theorem 4 it follows that $U(t)=V(t) T^{-1}$. Clearly, $U^{*}(t)=\left(T^{*}\right)^{-1} V^{*}(t)=$ $\left(T_{\#}\right)^{-1} V(-t)$. Since $U_{\#}(t)=V(-t) T_{\#}^{-1}$, it follows that

$$
U^{*}(t)=T_{\#}^{-1} U_{\#}(t) T_{\#} \text {. }
$$

We will need estimates on the gradients of $T f$, where $T$ is given by (3.1).

THEOREM 5. There is a constant $C$ that only depends on the Lipschitz constant of $\varphi$, such that if $\varphi$ is a test function then

$$
\int_{\mathbf{R}^{n-1}}|\nabla T f(x)|^{2} d x \leq C \int\left(|\nabla f(x)|^{2}+\left|\nabla_{2} \varphi(x)\right|^{2} f^{2}(x)\right) d x .
$$

Here $\nabla_{2} \varphi$ denotes the second gradient of $\varphi$, i.e., the vector of second order derivatives.

The proof of Theorem 5 will be based on the method of rotation and the following lemma.

LEMMA 1. Let $\varphi: \mathbf{R}^{d+1} \rightarrow \mathbf{R}$ be a test function. We define the operator $U: L^{2}\left(\mathbf{R}^{d+1}\right) \rightarrow L^{2}\left(\mathbf{R}^{d+1}\right)$ by putting

$$
U f(x, y)=\lim _{\varepsilon \downarrow 0} \int_{\mathbf{R}} \frac{f(x, \eta) d \eta}{y-\eta+i(\varphi(x, y)-\varphi(x, \eta)+\varepsilon)} .
$$

Then

$$
\left.\int_{\mathbf{R}^{d+1}}|\nabla U f|^{2} d x d y \leq C \int_{\mathbf{R}^{d+1}}|\nabla f|^{2}+(f(x, y))^{2}\left|\nabla_{2} \varphi(x, y)\right|^{2}\right) d x d y,
$$

where $C$ only depends on the Lipschitz constant of $\varphi$.

ProOF. First let $d=0$ and let $M g$ denote $\left(1+i \varphi^{\prime}\right) g$. Then by integration of parts

$$
\frac{\partial U f(y)}{\partial y}=M \lim _{\varepsilon \downarrow 0} \int \frac{M^{-1} f(\eta) d \eta}{(y-\eta)+i(\varphi(y)-\varphi(\eta)+\varepsilon)},
$$

so by the boundedness of the Cauchy integral we get from (3.3) that

$$
\begin{aligned}
\iint_{\mathbf{R}^{d+1}} & \left(\frac{\partial U f(x, y)}{\partial y}\right)^{2} d x d y \\
& \leq C \iint_{\mathbf{R}^{d+1}}\left(\left(\frac{\partial f(x, y)}{\partial y}\right)^{2}+(f(x, y))^{2}\left(\frac{\partial \varphi(x, y)}{\partial y}\right)^{2}\right) d x d y .
\end{aligned}
$$

By a similar integration by parts we get the remaining estimates of the gradient of $U f$.

The result that we now need is the following lemma. 
LEMMA 2. Let $\varphi: \mathbf{R}^{d+1} \rightarrow \mathbf{R}$ be a test function with $|\nabla \varphi| \leq L$. Suppose $F$ is holomorphic in a neighbourhood of $\{x \in \mathbf{R}:|x| \leq L\}$ and define $U$ as

$$
U f(x, y)=p v \int_{-\infty}^{\infty} f(x, \eta)(y-\eta)^{-1} F(y-\eta \varphi(x, y)-\varphi(x, \eta)) d \eta .
$$

Then $U$ is bounded on $L^{2}\left(\mathbf{R}^{d+1}\right)$ with a norm that only depends on $F$ and $L$. Furthermore,

$$
\iint_{\mathbf{R}^{d+1}}|\nabla U f(x, y)|^{2} d x d y \leq C \iint_{\mathbf{R}^{d+1}}\left(|\nabla f(x, y)|^{2}+(f(x, y))^{2}\left|\nabla_{2} \varphi(x, y)\right|^{2}\right) d x d y
$$

where $C$ only depends on $L$ and $F$.

PROOF. A straightforward modification of the proof of Lemma 1 gives the proof.

PROOF OF THEOREM 5. Combining Lemma 2 with the method of rotation (see Fabes, Jodeit and Rivière $[\mathbf{F J R}]$ ) gives the proof of Theorem 5 . We now have that if

$$
B=A \int_{0}^{\infty}(t \pi)^{-1 / 2} S(t) d t,
$$

where $A$ is the generator of the Poisson semigroup, then

$$
B^{2}=A \text {. }
$$

We need the following a priori estimate of $B$.

THEOREM 6. Suppose $f \in L^{2}(\Gamma)$ is smooth and denote by $u$ the harmonic extension of $f$. Then there is a constant $C$ that can be taken to depend only on the Lipschitz constant of $\varphi$ such that

$$
\|B f\|_{L^{2}(\Gamma)}^{2} \leq C \int_{D}|\nabla u|^{2} d P .
$$

ProOF. Putting $v(x, y)=\int_{y}^{\infty} u(x, s)(s-y)^{-1 / 2} d s$, we have that $B f$ equals the boundary values of $w=\pi^{-1 / 2} \partial v / \partial y$. We also have that $v$ and $w$ are harmonic. To see this, it is enough to show that $v$ is harmonic. This holds since, integrating by parts twice,

and hence

$$
v(x, y)=\frac{4}{3} \int_{y}^{\infty}(s-y)^{3 / 2} \frac{\partial^{2} u}{\partial s^{2}}(x, s) d s
$$

$$
\frac{\partial^{2} v}{\partial y^{2}}=\int_{y}^{\infty}(s-y)^{-1 / 2} \frac{\partial^{2} u}{\partial s^{2}}(x, s) d s=-\sum_{j=1}^{n-1} \frac{\partial^{2} v}{\partial x_{j}^{2}} .
$$

It is straightforward to verify that if $\delta(p)=\operatorname{dist}(P, D)$ then

$$
\int_{D}(\delta(P))^{2}\left|\nabla_{2} u(P)\right|^{2} d P \leq C \int_{D}|\nabla u(P)|^{2} d P .
$$

See the argument in Stein [S, p. 216]. From Hardy's inequality it now follows (see [S, p. 272]) that

$$
\int_{D} \delta\left(\frac{\partial w}{\partial y}\right)^{2} d P=\int_{D} \delta\left(\frac{\partial^{2} v}{\partial y^{2}}\right)^{2} d P \leq C \int \delta(P)^{2}\left|\nabla_{2} u\right|^{2} d P \leq C \int_{D}|\nabla u|^{2} d P,
$$

which together with the arguments for (2.2) and Theorem 2 yield the theorem.

We can now establish our main estimate for the bilinear form $B_{A}(u)$. 
THEOREM 7. If $u$ is harmonic in $D$, then

$$
\left|B_{A}(u)\right| \leq C\left|\left\|A \left|\|\mid\| M(u) \|_{L^{2}(\Gamma)},\right.\right.\right.
$$

where $C$ only depends on the Lipschitz constant of $\varphi$.

ProOF. Let $\eta \in C_{0}^{\infty}\left(\mathbf{R}^{n}\right)$ be nonnegative with $\int_{\mathbf{R}^{n}} \eta d x=1$ and put $\varphi(x, t)=$ $c t+\int_{\mathbf{R}^{N}} \varphi(x-y) \eta_{t}(y) d y, t \geq 0$, where $c \geq 0$ is chosen so large that $\partial \varphi / \partial t \geq 1$. Here $c$ can be chosen to depend only on $\eta$ and the Lipschitz constant of $\varphi$. We put for $t>0, D_{t}=\{(x, y): y>\varphi(x, t)\}$ and $\Gamma_{t}=\partial D_{t}$. We find by a change of variables that

$$
B_{A}(u)=\int_{0}^{\infty} \int_{\Gamma_{t}} a \frac{\partial u}{\partial y} d \sigma_{t} d t
$$

where $|a| \leq C|A|$ and $|\nabla a| \leq C\left(\left|\nabla_{2} h\right||A|+|\nabla A|\right)$, where $h(x, y)$ is defined by $\varphi(x, h(x, y))=y$. Since $t\left|\nabla_{2} \varphi\right|^{2}$ is a Carleson measure, it follows that $\delta\left|\nabla_{2} h\right|^{2}$ is a Carleson measure on $D$. Here $\sigma_{t}$ is the surface measure on $\Gamma$. Now let $B_{t}$ be the square root of the generator of the Poisson semigroup for $D_{t}$.

$$
\int_{\Gamma_{t}} a \frac{\partial u}{\partial t} d \sigma_{t}=\left\langle a, B_{t}^{2} u\right\rangle_{L^{2}\left(\Gamma_{t}\right)}=\left\langle B_{t}^{*} a, B_{t} u\right\rangle_{L^{2}\left(\Gamma_{t}\right)}
$$

since

$$
\int_{0}^{\infty}\left\|B_{t} u\right\|_{L^{2}\left(\Gamma_{t}\right)}^{2} d t \leq C \int_{0}^{\infty} \int_{D_{t}}|\nabla u|^{2} d P \leq C \int_{D} \delta(P)|\nabla u|^{2}
$$

by Theorem 6 . Now define $v$ by putting $v(P)=T_{t} a(P)$ for $P \in \Gamma_{t}$, where $T_{t}$ is the boundary value of the double layer potential. By Theorem 5 and (3.2) it follows that

$$
\begin{aligned}
\int_{0}^{\infty} & \left\|B_{t}^{*} a\right\|_{L^{2}\left(\Gamma_{t}\right)}^{2} d t \leq C \int_{0}^{\infty} \int_{D_{t}}|\nabla v|^{2} d P \\
& \leq C \int_{0}^{\infty} \int_{D_{t}}\left(|A|^{2}\left|\nabla_{2} h\right|^{2}+C|\nabla A|^{2}\right) d P d t \\
& \leq C \int_{D} \delta\left(\left|\nabla_{2} h\right|^{2} A^{2}+C|\nabla A|^{2}\right) \leq C|\|A\||^{2},
\end{aligned}
$$

which completes the proof of Theorem 7 . We remark that it is now straightforward to deduce (1.7) from Theorem 7 by writing $f \in L^{2}(\partial D)$ as a sum of functions with small supports and then argue as, e.g., in [DK].

We finally remark that estimate (1.7) does not hold for general functions $u$ and $A$ for which $\||A|\|<\infty$ and $\||u|\|<\infty$. Let $\eta \geq 0$ be a test function and put $A(x, y)=\eta(x, y) \sin \left(|\log y|^{1 / 2-\varepsilon}\right)$ and $u(x, y)=\eta(x, y) \cos \left(|\log y|^{1 / 2-\varepsilon}\right)$. If $0<$ $\varepsilon<1 / 2$, then $\left\|\left|u_{\nu}\right|\right\|||\left|A_{\nu}\right| \| \leq C$ independent of $\nu$, where $u_{\nu}(x, y)=u(x, y+1 / \nu)$, $\nu=1,2, \ldots$. However, it is easily seen that

$$
\lim _{\nu \rightarrow \infty}\left|\int_{y>0} \int_{x} A_{\nu}(x, y) \frac{\partial u_{\nu}(x, y)}{\partial y} d x d y\right|=\infty .
$$

\section{REFERENCES}

[C] A. P. Calderon, Commutators, singular integrals on Lipschitz curves and applications, Proc. Internat. Congress Math. ICM, Helsinki, 1980, pp. 85-95.

[CMM] R. R. Coifman, A. McIntosh and Y. Meyer, L'intégrale de Cauchy définit un opérateur borné sur $L^{2}$ pour les courbes Lipschitz innes, Ann. of Math. (2) 116 (1982), 361-387. 
[D1] B. E. J. Dahlberg, On estimates of harmonic measures, Arch. Rational Mech. Anal. 65 (1977), 272-288.

[D2] _ Weighted norm inequalities for the Lusin area integral and the nontangential maximal function for functions harmonic in a Lipschitz domain, Studia Math. 67 (1980), 297-314.

[D3] _ On the absolute continuity of elliptic measures, Preprint, Dept. of Math., Chalmers Univ. of Technology and Univ. of Göteborg, 1984-28.

[D4] _ Real analysis and potential theory, Proc. Internat. Congress Math. Warsaw, 1983.

[DK] B. E. J. Dahlberg and C. E. Kenig, Hardy spaces and the $L^{p}$-Neumann problem for Laplace's equation in a Lipschitz domain, Ann. of Math. (to appear).

[DKV] B. E. J. Dahlberg, C. E. Kenig and G. C. Verchota, The Dirichlet problem for the biharmonic equation in a Lipschitz domain (to appear).

[FJR] E. Fabes, M. Jodeit and N. Rivière, Potential techniques for boundary value problems on $C^{1}$-domains, Acta Math. 141 (1978), 165-186.

[JK] D. S. Jerison and C. E. Kenig, The Neumann problems on Lipschitz domains, Bull. Amer. Math. Soc. (N.S.) 4 (1981), 203-207.

[K] C. E. Kenig, Recent progress on boundary value problems on Lipschitz domains, Univ. of Minnesota, Math. Report 84-133.

[M] Y. Meyer, Regularité des solutions des equations aux dérivées partielles non linéaires, Sém. Bourbaki, Vol. 1979/80, Exp. 543-560, Lecture Notes in Math., Vol. 842, SpringerVerlag, Berlin and New York, 1981, pp. 293-302.

[S] E. M. Stein, Singular integrals and differentiability properties of functions, Princeton Univ. Press, Princeton, N. J., 1970.

[V] G. C. Verchota, Layer potentials and boundary value problems for Laplace's equation in Lipschitz domains, Thesis, Univ. of Minnesota, 1982; J. Funct. Anal. (to appear).

[Y] K. Yoshida, Functional analysis, Springer-Verlag, Berlin and New York, 1978.

Department of Mathematics, Chalmers University of Technology and the UNIVERSITY OF GÖTEBORG, GÖTEBORG, SWEDEN 